\title{
Global spread and impacts of emerging vector-borne diseases
}

\author{
Kulkarni MA ${ }^{1 *}$ \\ Affiliation \\ ${ }^{1}$ School of Epidemiology, Public Health \& Preventive Medicine, University of Ottawa, Ottawa, ON \\ ^Correspondence: manisha.kulkarni@uottawa.ca
}

Suggested citation: Kulkarni MA. Global spread and impacts of emerging vector-borne diseases. Can Comm Dis Rep 2016;42:198-9. https://doi.org/10.14745/ccdr.v42i10a02

\section{Background}

With increasing globalization, new and emerging infectious diseases are becoming worldwide health priorities. Vector-borne diseases (VBDs), in particular those transmitted by mosquitoes, comprise a significant proportion of recent emerging infectious disease events. Factors such as climate change, pathogen evolution, deteriorating vector control and sociodemographic and environmental changes, in the context of population growth and often rapid, uncontrolled urbanization, may all facilitate the geographic spread of infections, including mosquito-borne diseases.

\section{Objective}

To describe the recent global trends and impacts of emerging VBDs and review the factors that have contributed to their emergence and spread.

\section{Narrative}

The explosive emergence of Zika virus in the Americas in the past year, following its expansion across Africa and Asia, highlights a rising trend in the global spread of mosquito-borne diseases. Of recent concern is the spread of viruses transmitted by aedes mosquitoes, including dengue, chikungunya and Zika viruses. These infections pose a particular threat to urban populations, most notably in low- and middle-income countries that are experiencing rapid rates of urbanization. High population densities, and a lack of adequate housing and sanitation, promote the breeding of Aedes aegypti and Aedes albopictus mosquito species in containers and debris commonly found near houses.

Dengue, chikungunya and Zika viruses share the same vectors and similar ecological origins, so the co-circulation of these viruses in many parts of the world is not unexpected. In endemic areas of Africa and Asia, the viruses are maintained in sylvatic cycles where they circulate between wildlife reservoir hosts and mosquito vectors. Spillover to human populations commonly occurs when people encroach on sylvatic cycles, which can lead to the virus circulating in urban mosquito populations and, ultimately, to major outbreaks of disease (1). In turn, urban disease outbreaks in immunologically naïve populations result in high rates of morbidity and mortality, particularly in areas with limited vector surveillance and control resources. The movement of people and goods through international travel and trade may drive the spread of mosquito-borne viruses to regions of the world where competent vectors exist. This trend is further exacerbated by climate change, which is increasing local suitability for disease transmission in many previously non-endemic regions.

The global spread of Zika virus exemplifies key factors that may drive the emergence and expansion of VBDs on a global scale. First identified in Uganda in 1947, Zika virus was reported to cause sporadic mild illness in equatorial Africa and parts of Asia (an outbreak occurred in Micronesia in 2007). Subsequently, the virus spread across the Pacific region, causing a large outbreak in French Polynesia in 2013; it was first detected in the Americas in 2014 (2). Since its emergence in Brazil, with the first cases reported in early 2015, Zika virus has spread to at least 35 countries in the Americas, causing an unknown number of infections and thousands of locally acquired cases of microcephaly and Guillain-Barré syndrome. Cases of sexually transmitted infections have now been documented in countries without local transmission. The spread of Zika has been attributed to the movement of viremic travellers from Brazil to regions with established Ae. aegypti mosquito populations (3). In addition, record high temperatures and severe drought conditions across north and eastern South America, associated with the recent El Niño, may have contributed to the rapid emergence of Zika in 2015 (4). Furthermore, molecular changes have been noted in the Asian lineage of Zika virus that may be associated with the observed increase in pathogenicity and transmissibility (5).

\section{Conclusion}

The worldwide emergence and spread of VBDs is being driven by recent global changes. In addition to increased public health response, a better understanding of the epidemiology of VBDs is needed to identify the drivers of these epidemics and inform the public health response. These outbreaks are the sources of new pathogens that may arrive in Canada and elsewhere. Given the importance of VBDs to global health and Canada's vulnerability with respect to vector-disease emergence, there is an important and urgent need to strengthen global capacity 
in VBD research, surveillance, prevention and response. It is important to note that the emergence and expansion of diseases in resource-limited countries with weak surveillance capacity and health systems increases the risk of introducing disease to other countries because of increasing global interconnectedness. This highlights the need for coordination and global health partnerships to address emerging VBDs in Canada and abroad.

\section{Conflict of interest}

None.

\section{References}

1. Weaver SC. Urbanization and geographic expansion of zoonotic arboviral diseases: Mechanisms and potential strategies for prevention. Trends Microbiol 2013;21:360-3.

2. Kindhauser MK, Allen T, Frank V, Santhana RS, Dye C. Zika: the origin and spread of a mosquito-borne virus. Bull World Health Organ 2016;1-18.

3. Bogoch II, Brady OJ, Kraemer MU, German M, Creatore MI Kulkarni MA et al. Anticipating the international spread of Zika virus from Brazil. Lancet 2016;387:335-6.

4. Paz S, Semenza JC. El Niño and climate changecontributing factors in the dispersal of Zika virus in the Americas? Lancet 2016; 387:745.

5. Wang L, Valderramos SG, Wu A, Ouyang S, Li C, Brasil P et al. From Mosquitos to Humans: Genetic Evolution of Zika Virus. Cell Host Microbe 2016;19(5):561-5.

\section{Would you like to publish in CCDR?}

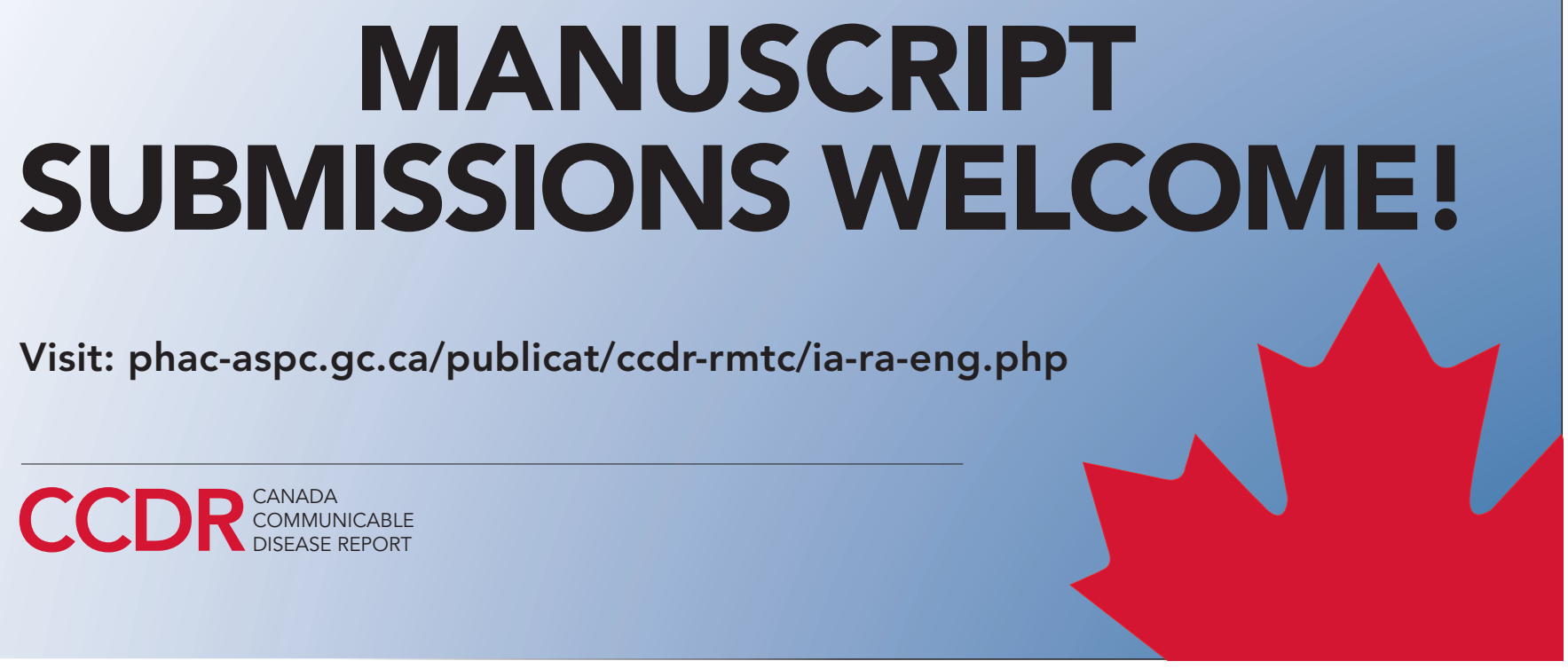

